\title{
Ground state and low excitations of an integrable chain with alternating spins
}

\author{
St Meißner‡ and B - D Dörfel§ \\ Institut für Physik, Humboldt-Universität, Theorie der Elementarteilchen \\ Invalidenstraße 110, 10115 Berlin, Germany
}

\begin{abstract}
An anisotropic integrable spin chain, consisting of spins $s=1$ and $s=\frac{1}{2}$, is investigated [1]. It is characterized by two real parameters $\bar{c}$ and $\tilde{c}$, the coupling constants of the spin interactions. For the case $\bar{c}<0$ and $\tilde{c}<0$ the ground state configuration is obtained by means of thermodynamic Bethe ansatz. Furthermore the low excitations are calculated. It turns out, that apart from free magnon states being the holes in the ground state rapidity distribution, there exist bound states given by special string solutions of Bethe ansatz equations (BAE) in analogy to [13]. The dispersion law of these excitations is calculated numerically.
\end{abstract}

PACS numbers: $75.10 \mathrm{JM}, 75.40 \mathrm{Fa}$

Short title: $\mathrm{XXZ}\left(\frac{1}{2}, 1\right)$ Heisenberg spin chain

April 26, 2018

$\ddagger$ email: meissner@qft2.physik.hu-berlin.de

$\S$ email: doerfel@ifh.de 


\section{Introduction}

Since the development of the quantum inverse scattering method (QISM) [2, 3] many

generalisations of the well known XXZ $\left(\frac{1}{2}\right)$ model have been investigated [4, 5, 6]. The appearance of interesting new features of these models made those analysises very fruitful.

In 1992 de Vega and Woynarovich suggested a new kind of generalisation by constructing a model containing spin- $\frac{1}{2}$ - and spin-1-particles [四]. In comparison with models containing only one kind of spin it shows a richer structure due to additional parameters, the spin interaction coupling constants, making an investigation worthwhile.

Its isotropic limit $\operatorname{XXX}\left(\frac{1}{2}, 1\right)$ has been studied in papers [14, 15]. The generalisation procedure was extended to pairs of higher spins $\left(\frac{1}{2}, S\right)$ and $\left(S^{\prime}, S\right)$ in [16, 17, 18]. Except [14] only the conformally invariant case with positive coupling was considered. Our aim therefore is to go beyond that point. We intend to continue along this line in a subsequent publication.

In this paper we want to study the $\mathrm{XXZ}\left(\frac{1}{2}, 1\right)$ model with strictly alternating spins. Definitions are reviewed in section 2 . In section 3 we carry out the thermodynamic Bethe ansatz (TBA) and obtain the ground state for negative coupling of the spin interactions. Section 4 deals with the higher level Bethe ansatz for low excitations above this ground state and section 5 contains our conclusions.

\section{Description of the model}

We consider the Hamiltonian of a spin chain of length $2 N$ [1]

$$
\mathcal{H}(\gamma)=\bar{c} \overline{\mathcal{H}}(\gamma)+\tilde{c} \tilde{\mathcal{H}}(\gamma)-H S^{z}
$$

where $\overline{\mathcal{H}}$ couples two spins $s=\frac{1}{2}$ and one spin $s=1$, while the converse is true for $\tilde{\mathcal{H}}$ (for explicit construction see again [1], bars and tildes are interchanged with respect to this reference as in subsequent publications of de Vega et al.). $H$ is an external magnetic field. We impose periodic boundary conditions.

The Hamiltonian contains an XXZ type anisotropy, which is parametrized by $e^{i \gamma}$ or $e^{-\gamma}$ respectively. We investigate the weak antiferromagnetic case, i.e. the parametrization is $e^{i \gamma}$ and we restrict ourselves to $0<\gamma<\pi / 2$. Moreover we have two additional real parameters $\tilde{c}$ and $\bar{c}$ being the coupling constants of the spin interactions and dominating the qualitative behaviour of the model.

The Bethe ansatz equations (BAE) determining the solution of the model are

$$
\left(\frac{\sinh \left(\lambda_{j}+i \frac{\gamma}{2}\right)}{\sinh \left(\lambda_{j}-i \frac{\gamma}{2}\right)} \frac{\sinh \left(\lambda_{j}+i \gamma\right)}{\sinh \left(\lambda_{j}-i \gamma\right)}\right)^{N}=-\prod_{k=1}^{M} \frac{\sinh \left(\lambda_{j}-\lambda_{k}+i \gamma\right)}{\sinh \left(\lambda_{j}-\lambda_{k}-i \gamma\right)}, \quad j=1 \ldots M
$$


One can express energy, momentum and spin in terms of BAE roots $\lambda_{j}$ :

$$
\begin{aligned}
& E=\bar{c} \bar{E}+\tilde{c} \tilde{E}-\left(\frac{3 N}{2}-M\right) H, \\
& \bar{E}=-\sum_{j=1}^{M} \frac{2 \sin \gamma}{\cosh 2 \lambda_{j}-\cos \gamma}, \\
& \tilde{E}=-\sum_{j=1}^{M} \frac{2 \sin 2 \gamma}{\cosh 2 \lambda_{j}-\cos 2 \gamma}, \\
& P=\frac{i}{2} \sum_{j=1}^{M}\left\{\log \left(\frac{\sinh \left(\lambda_{j}+i \frac{\gamma}{2}\right)}{\sinh \left(\lambda_{j}-i \frac{\gamma}{2}\right)}\right)+\log \left(\frac{\sinh \left(\lambda_{j}+i \gamma\right)}{\sinh \left(\lambda_{j}-i \gamma\right)}\right)\right\}, \\
& S_{z}=\frac{3 N}{2}-M .
\end{aligned}
$$

Here we have substracted a constant in the momentum in order to make this magnitude vanishing for the ferromagnetic state. Furthermore additive constants in $\overline{\mathcal{H}}$ and $\tilde{\mathcal{H}}$ are dropped due to different normalisation compared with [1].

\section{Thermodynamic Bethe ansatz (TBA) and ground state for negative coupling constants}

We want to study the model in the thermodynamic limit $N \rightarrow \infty$. Then only solutions of the string type occur (for $M$ fix):

$$
\lambda_{\alpha}^{n, j, \nu}=\lambda_{\alpha}^{n, \nu}+i(n+1-2 j) \frac{\gamma}{2}+\frac{1}{4} i \pi(1-\nu)+\delta_{\alpha}^{n, j, \nu}, \quad j=1 \ldots n .
$$

Here $\lambda_{\alpha}^{n, \nu}$ is the real center of the string, $n$ is the string length and $\nu$ the parity of the string with values \pm 1 . The last term is a correction due to finite size effects.

Now the permitted values of the string length $n$ and related parities $\nu$ depending on the anisotropy $\gamma$ are to determine. Several approaches have been used to obtain them for the massive Thirring model [8] and for the $\mathrm{XXZ}\left(\frac{1}{2}\right)$ [7] and $\mathrm{XXZ}(\mathrm{S})$ models [10, 11, 12] all leading to the Takahashi conditions, a system of inequalities describing admissible string length and parities:

$$
\nu_{n} \sin \gamma j \sin \gamma(n-j)>0, \quad j=1 \ldots n-1 .
$$

In the XXZ(S) case additional restrictions on the spin arise.

By applying similar methods to our model one can show that fulfilling the Takahashi conditions is necessary and sufficient for an admissible pair $\left(n, \nu_{n}\right)$ for the number of magnons $M$ fix. On the other hand we did not succeed in deriving such conditions in the case $N, M \rightarrow \infty, M / N$ fix from the BAE directly. Nevertheless there is a series of 
arguments favouring the conjecture that in this case the same strings as in the previous one can exist. In the following we want to state a few of them briefly.

Generally the string hypothesis is believed to be valid in the case $N, M \rightarrow \infty, M / N$ fix (i.e. string corrections are exponentially small) for non-vanishing external magnetic field (see e.g. [9] and [15]). For zero magnetic field the numerical results of Frahm et al. show that string corrections are of orders $O(1 / N)$ to $O(1)$, what does not affect the thermodynamics of the model.

For further discussion it is important, that, as already proven in [1], the 'sea-strings' are exponentially exact.

Furthermore Takahashi and Suzuki obtained a phenomenological rule determining admissible strings for the $\operatorname{XXZ}\left(\frac{1}{2}\right)$ model proven to be equivalent to the Takahashi conditions. Fulfilling this criterion requires that a singlet state can be constructed by introducing a maximum number of strings of one type. Kirillov and Reshetikhin generalized this criterion naturally to higher spins. In the $\mathrm{XXZ}(\mathrm{S})$ it is as well a selection rule for permitted spin values as a criterion for an admissible pair $\left(n, \nu_{n}\right)$.

In the following we therefore shall assume the existence of strings fulfilling the Takahashi conditions.

Substituting (3.1) into (2.2) and taking the logarithm yields

$$
N t_{j, 1}\left(\lambda_{\alpha}^{n_{j}}\right)+N t_{j, 2}\left(\lambda_{\alpha}^{n_{j}}\right)=2 \pi I_{\alpha}^{n_{j}}+\sum_{k} \sum_{\beta} \Theta_{j k}\left(\lambda_{\alpha}^{n_{j}}-\lambda_{\beta}^{n_{k}}, \nu_{j} \nu_{k}\right)
$$

with the known notations

$$
\begin{gathered}
t_{j, 2 S}(\lambda)=\sum_{k=1}^{\min \left(n_{j}, 2 S\right)} f\left(\lambda,\left|n_{j}-2 S\right|+2 k-1, \nu_{j}\right), \\
\Theta_{j k}(\lambda)=f\left(\lambda,\left|n_{j}-n_{k}\right|, \nu_{j} \nu_{k}\right) \\
+f\left(\lambda,\left(n_{j}+n_{k}\right), \nu_{j} \nu_{k}\right)+2 \sum_{k=1}^{\min \left(n_{j}, n_{k}\right)-1} f\left(\lambda,\left|n_{j}-n_{k}\right|+2 k, \nu_{j} \nu_{k}\right),
\end{gathered}
$$

and

$$
f(\lambda, n, \nu)=\left\{\begin{array}{rl}
0 & n \gamma / \pi \in \mathbf{Z} \\
2 \nu \arctan \left((\cot (n \gamma / 2))^{\nu} \tanh \lambda\right) & n \gamma / \pi \notin \mathbf{Z}
\end{array} .\right.
$$

Here we have used that a given string length $n>1$ corresponds to a unique parity, what is a consequence of (3.2). The numbers $I_{\alpha}^{n_{j}}$ are half-odd-integers counting the strings of length $n_{j}$.

Introducing particle and hole densities in the usual way (see e.g. [5] eqs. 60-63) we perform the limiting process $N \rightarrow \infty$

$$
a_{j, 1}(\lambda)+a_{j, 2}(\lambda)=\left(\rho_{j}(\lambda)+\tilde{\rho}_{j}(\lambda)\right)(-1)^{r(j)}+\sum_{k} T_{j k} * \rho_{k}(\lambda),
$$


where $a * b(\lambda)$ denotes the convolution

$$
a * b(\lambda)=\int_{-\infty}^{\infty} d \mu a(\lambda-\mu) b(\mu)
$$

and

$$
a_{j, 2 S}(\lambda)=\frac{1}{2 \pi} \frac{d}{d \lambda} t_{j, 2 S}(\lambda), \quad T_{j, k}(\lambda)=\frac{1}{2 \pi} \frac{d}{d \lambda} \Theta_{j, k}(\lambda) .
$$

The sign $(-1)^{r(j)}$ results from the requirement of positive densities in the 'noninteracting' limit (i.e. only one string type is present)[12].

The analysis of (3.2) remains complicated for arbitrary $\gamma$ [7], but at special values the picture becomes easy, namely $\gamma=\pi / \mu, \quad \mu \ldots$ integer, $\mu \geq 3$. Following Babujian and Tsvelick [6] we want to consider that case only.

Due to periodicity we are left with strings

(i) $n_{j}=j, \quad j=1 \ldots \mu-1, \quad \nu_{j}=1$

(ii) $n_{\mu}=1, \quad \nu_{\mu}=-1$.

Equation (3.7) then reduces to

$$
\begin{aligned}
& a_{j, 1}(\lambda)+a_{j, 2}(\lambda)=\rho_{j}(\lambda)+\tilde{\rho}_{j}(\lambda)+\sum_{k=1}^{\mu} T_{j k} * \rho_{k}(\lambda), \quad j=1 \ldots \mu-1, \\
& a_{\mu, 1}(\lambda)+a_{\mu, 2}(\lambda)=-\rho_{\mu}(\lambda)-\tilde{\rho}_{\mu}(\lambda)+\sum_{k=1}^{\mu} T_{\mu k} * \rho_{k}(\lambda) .
\end{aligned}
$$

We are now able to express energy, momentum and spin in terms of the densities via (2.3), (2.4) and (2.5). The standard procedure leads to equations determining the equilibrium state at temperature $T$ :

$T \ln \left(1+\exp \left(\frac{\epsilon_{j}}{T}\right)\right)=$

$$
H n_{j}-2 \pi \bar{c} a_{j, 1}(\lambda)-2 \pi \tilde{c} a_{j, 2}(\lambda)+\sum_{k=1}^{\mu} T \ln \left(1+\exp \left(\frac{-\epsilon_{k}}{T}\right)\right) * A_{j k}(\lambda)
$$

with

$$
A_{j k}(\lambda)=(-1)^{r(k)} T_{j k}\left(\lambda, \nu_{j} \nu_{k}\right)+\delta(\lambda) \delta_{j k}
$$

and

$$
\frac{\tilde{\rho}_{j}}{\rho_{j}}=\exp \left(\frac{\epsilon_{j}}{T}\right)
$$

Again the free energy can be expressed in terms of our new variables $\epsilon_{j}(\lambda)$ :

$2 \mathcal{F}=\frac{F}{N}=-\int_{-\infty}^{\infty} d \lambda \sum_{j=1}^{\mu}(-1)^{r(j)}\left(a_{j, 1}(\lambda)+a_{j, 2}(\lambda)\right) T \ln \left(1+\exp \left(\frac{-\epsilon_{j}}{T}\right)\right)-\frac{3 H}{2}$. 
Reversing the operator $A_{j k}$ in (3.11) by applying

$$
C_{j k}=\delta(\lambda) \delta_{j k}-s(\lambda)\left(\delta_{j+1 k}+\delta_{j-1 k}\right), \quad j, k=1 \ldots \mu
$$

with

$$
s(\lambda)=\frac{1}{2 \gamma \cosh (\pi \lambda / \gamma)}
$$

yields

$$
\begin{aligned}
\epsilon_{1}(\lambda) & =T s * \ln \left(f\left(\epsilon_{2}\right)\right)-2 \pi \bar{c} s(\lambda) \\
\epsilon_{2}(\lambda) & =T s * \ln \left(f\left(\epsilon_{3}\right) f\left(\epsilon_{1}\right)\right)-2 \pi \tilde{c} s(\lambda) \\
\epsilon_{j}(\lambda) & =T s * \ln \left(f\left(\epsilon_{j+1}\right) f\left(\epsilon_{j-1}\right)\right)+\delta_{j \mu-2} T s * \ln \left(f\left(-\epsilon_{\mu}\right)\right), j=3 \ldots \mu-2 \\
\epsilon_{\mu-1}(\lambda) & =\frac{H \mu}{2}+T s * \ln \left(f\left(\epsilon_{\mu-2}\right)\right) \\
\epsilon_{\mu}(\lambda) & =\frac{H \mu}{2}-T s * \ln \left(f\left(\epsilon_{\mu-2}\right)\right)
\end{aligned}
$$

with

$$
f(x)=1+e^{x / T}
$$

From these equations it can be easily established that $\epsilon_{j} \geq 0$ for $j=3 \ldots \mu-1$.

Since we are interested in the ground state we take the limit $T \rightarrow 0$ in equations (3.11) and (3.14). Taking into account that $\epsilon_{j} \geq 0$ for $j=3 \ldots \mu-1$ we have

$$
\begin{aligned}
& \epsilon_{j}^{+}(\lambda)=H n_{j}-2 \pi \bar{c} a_{j, 1}(\lambda)-2 \pi \tilde{c} a_{j, 2}(\lambda)-\epsilon_{1}^{-} * A_{j 1}(\lambda)-\epsilon_{2}^{-} * A_{j 2}(\lambda)-\epsilon_{\mu}^{-} * A_{j \mu}(\lambda) \\
& \begin{aligned}
2 \mathcal{F}=\frac{F}{N}= & \int_{-\infty}^{\infty} d \lambda\left[\left(a_{1,1}(\lambda)+a_{1,2}(\lambda)\right) \epsilon_{1}^{-}(\lambda)\right. \\
& \left.\quad+\left(a_{2,1}(\lambda)+a_{2,2}(\lambda)\right) \epsilon_{2}^{-}(\lambda)-\left(a_{\mu, 1}(\lambda)+a_{\mu, 2}(\lambda)\right) \epsilon_{\mu}^{-}(\lambda)\right]-\frac{3 H}{2}
\end{aligned}
\end{aligned}
$$

where $\epsilon_{j}^{+}$and $\epsilon_{j}^{-}$denote positive and negative parts of the function $\epsilon_{j}$ respectively.

Now we want to discuss equation (3.19) for the case $H=0^{+}$in dependence of the signs of $\bar{c}$ and $\tilde{c}$.

(i) $\bar{c}>0, \tilde{c}>0$

This sector has been investigated completely by de Vega and Woynarovich [1]. The solution is

$$
\epsilon_{j}(\lambda)=-2 \pi \bar{c} s(\lambda) \delta_{j 1}-2 \pi \tilde{c} s(\lambda) \delta_{j 2}
$$

(ii) $\bar{c}>0, \tilde{c}<0$

The picture in this sector is difficult, since the quadrant is divided by a phase line starting in the origin and going to infinity, which we obtained from the TBA 
equations. In the lower area the model behaves as in case (iv), while in the upper one we expect for the ground state a mixture of strings of length 1 with different parities and finite Fermi zones. We hope to return to this case in greater detail later.

(iii) $\bar{c}<0, \tilde{c}>0$

The situation in this sector is similar to the case above. Again we have a phase line producing one area of case (iv) type and another one where the ground state is expected to be formed by the $(2,+)$ - and $(1,-)$-strings having again finite Fermi zones.

(iv) $\bar{c} \leq 0, \tilde{c} \leq 0$

In this case $\epsilon_{1}(\lambda)$ and $\epsilon_{2}(\lambda)$ are positive. The only string present in the ground state is $(1,-)$. Equations (3.19) and (3.20) reduce to

$$
\begin{aligned}
& \epsilon_{j}^{+}(\lambda)=-2 \pi \bar{c} a_{j, 1}(\lambda)-2 \pi \tilde{c} a_{j, 2}(\lambda)-\epsilon_{\mu}^{-} * A_{j \mu}(\lambda), \\
& 2 \mathcal{F}=\frac{F}{N}=-\int_{-\infty}^{\infty} d \lambda\left(a_{\mu, 1}(\lambda)+a_{\mu, 2}(\lambda)\right) \epsilon_{\mu}^{-}(\lambda) .
\end{aligned}
$$

The solutions of (3.22) can be given via their Fourier transforms:

$$
\begin{aligned}
& \hat{\epsilon}_{1}(p)=-2 \pi \bar{c} \frac{\cosh [p \gamma(\mu-2) / 2]}{\cosh [p \gamma(\mu-1) / 2]}-2 \pi \tilde{c} \frac{\cosh [p \gamma(\mu-3) / 2]}{\cosh [p \gamma(\mu-1) / 2]}, \\
& \hat{\epsilon}_{j}(p)=-2 \pi \bar{c} \frac{\cosh [p \gamma(\mu-j-1) / 2]}{\cosh [p \gamma(\mu-1) / 2]}-2 \pi \tilde{c} \frac{2 \cosh (p \gamma / 2) \cosh [p \gamma(\mu-j-1) / 2]}{\cosh [p \gamma(\mu-1) / 2]}, \\
& \hat{\epsilon}_{\mu-1}(p)=-2 \pi \bar{c} \frac{1}{2 \cosh [p \gamma(\mu-1) / 2]}-2 \pi \tilde{c} \frac{\cosh (p \gamma / 2)}{\cosh [p \gamma(\mu-1) / 2]}, \\
& \hat{\epsilon}_{\mu}(p)=2 \pi \bar{c} \frac{1}{2 \cosh [p \gamma(\mu-1) / 2]}+2 \pi \tilde{c} \frac{\cosh (p \gamma / 2)}{\cosh [p \gamma(\mu-1) / 2]} .
\end{aligned}
$$

The ground state energy is

$$
\begin{aligned}
2 \mathcal{F}=\frac{F}{N}=\bar{c} & \int_{-\infty}^{\infty} d p \frac{\sinh (p \gamma / 2)+\sinh (p \gamma)}{2 \cosh [p \gamma(\mu-1) / 2] \sinh (p \gamma \mu / 2)} \\
& +\tilde{c} \int_{-\infty}^{\infty} d p \frac{[\sinh (p \gamma / 2)+\sinh (p \gamma)] \cosh (p \gamma / 2)}{\cosh [p \gamma(\mu-1) / 2] \sinh (p \gamma \mu / 2)} .
\end{aligned}
$$

For $\bar{c}=\tilde{c}$ the continuum limit provides a conformal invariant theory. Due to the existence of one type of elementary excitations the central charge of the Virasoro algebra is $c=1$. That is remarkable, because in sector (i) $c=2$ [1]. The point $\bar{c}=\tilde{c}=0$ must be singular then, when passed on the conformal line.

(v) $\bar{c}=0, \tilde{c}>0$ 
(vi) $\bar{c}>0, \tilde{c}=0$

Both cases have been briefly considered in [14]. The two lines are infinitely high singular, because of the infinite degeneration of the ground state.

In the following we shall consider the case (iv) only.

\section{Higher level Bethe ansatz for low excitations}

In this section we want to derive equations for excitations above the ground state in the case $\bar{c}<0, \tilde{c}<0$. Starting point of our analysis is the result of section 3 . Though we have derived the ground state configuration of BAE roots only for the special case $\gamma=\pi / \mu, \mu$ integer, we extend this result to the whole range of $\gamma, 0<\gamma<\pi / 2$, motivated by the results of Frahm et al. [12], who found for the XXZ(S) Hamiltonians, where only special intervals of the anisotropy $\gamma$ are permitted, that the ground state configuration does not change within any of these intervals. Since in our case no restrictions on $\gamma$ arise, i.e. we have only one permitted interval, we expect the ground state configuration to be the one obtained for the above mentioned special values in the whole $\gamma$-range.

We write down the BAE for this ground state in the limit $N \rightarrow \infty$ with a finite number of excitations (holes in the ground state rapidity distribution and additional complex roots):

$$
\begin{aligned}
\bar{\phi}^{\prime}(\lambda, \gamma / 2)+\bar{\phi}^{\prime}(\lambda, \gamma) & =-\rho(\lambda)-\frac{1}{N} \sum_{h=1}^{N_{h}} \delta\left(\lambda-\lambda_{h}\right) \\
& +\int_{-\infty}^{\infty} d \lambda^{\prime} \rho\left(\lambda^{\prime}\right) \phi^{\prime}\left(\lambda-\lambda^{\prime}, \gamma\right)+\frac{1}{N} \sum_{l=1}^{N_{l}} \bar{\phi}^{\prime}\left(\lambda-z_{l}, \gamma\right)
\end{aligned}
$$

Here we have introduced the new notations

$$
\phi\left(\lambda, \frac{n \gamma}{2}\right)=\frac{1}{2 \pi} f(\lambda, n,+1), \quad \bar{\phi}\left(\lambda, \frac{n \gamma}{2}\right)=\frac{1}{2 \pi} f(\lambda, n,-1)
$$

and the prime means the derivative with respect to the first argument. The numbers of holes $\lambda_{h}$ and additional complex roots $z_{l}$ are denoted by $N_{h}$ and $N_{l}$ respectively. The hole positions are defined as solutions of (3.3) for the omitted $I_{h}$.

The solution of (4.1) contains different contributions obtained by Fourier transformation:

$$
\rho(\lambda)=\rho_{0}(\lambda)+\frac{1}{N}\left(\rho_{h}(\lambda)+\rho_{c}(\lambda)+\rho_{w}(\lambda)\right)
$$

with

$$
\begin{aligned}
& \hat{\rho_{0}}(p)=\frac{1+2 \cosh (p \gamma / 2)}{2 \cosh (p(\pi-\gamma) / 2)} \\
& \hat{\rho_{h}}(p)=-\sum_{h=1}^{N_{h}} \frac{e^{-i p \lambda_{h}} \sinh (p \pi / 2)}{2 \sinh (p \gamma / 2) \cosh (p(\pi-\gamma) / 2)}
\end{aligned}
$$




$$
\begin{aligned}
& \hat{\rho_{c}}(p)=-\sum_{l=1}^{N_{c} / 2} e^{-i p \sigma_{l}} \frac{\cosh (p \gamma / 2) \cosh \left(p \tau_{l}\right)}{\cosh (p(\pi-\gamma) / 2)} \\
& \hat{\rho_{w}}(p)=\sum_{l=1}^{N_{w} / 2} e^{-i p \sigma_{l}} \frac{\sinh (p(\pi-\gamma) / 2) \cosh \left(p\left(\pi-2 \tau_{l}\right) / 2\right)}{\cosh (p(\pi-\gamma) / 2) \sinh (p \gamma / 2)} .
\end{aligned}
$$

Here we have distinguished between complex close $\left(\left|\operatorname{Im}\left(z_{l}\right)\right|<\pi / 2-\gamma\right)$ and wide roots $\left(\left|\operatorname{Im}\left(z_{l}\right)\right|>\pi / 2-\gamma\right)$ differing in their Fourier transforms. Moreover we have used that complex roots appear in conjugated pairs $\left(z_{l}, \bar{z}_{l}\right)$ only.

Energy and momentum of the elementary hole excitations are given through

$$
\begin{aligned}
& \varepsilon_{h}\left(\lambda_{h}\right)=\bar{\varepsilon}_{h}\left(\lambda_{h}\right)+\tilde{\varepsilon}_{h}\left(\lambda_{h}\right) \\
& \bar{\varepsilon}_{h}\left(\lambda_{h}\right)=-\frac{\pi \bar{c}}{\pi-\gamma} \frac{1}{\cosh \left(\pi \lambda_{h} /(\pi-\gamma)\right)}, \\
& \tilde{\varepsilon}_{h}\left(\lambda_{h}\right)=-\frac{4 \pi \tilde{c}}{\pi-\gamma} \frac{\cos (\pi \gamma / 2(\pi-\gamma)) \cosh \left(\pi \lambda_{h} /(\pi-\gamma)\right)}{\cosh \left(2 \pi \lambda_{h} /(\pi-\gamma)\right)+\cos (\pi \gamma /(\pi-\gamma))}, \\
& p_{h}\left(\lambda_{h}\right)=\frac{1}{2} \arctan \left(\sinh \left(\frac{\pi \lambda_{h}}{\pi-\gamma}\right)\right)+\frac{\pi}{4}+\arctan \left(\frac{\sinh \left(\pi \lambda_{h} /(\pi-\gamma)\right)}{\cos (\pi \gamma / 2(\pi-\gamma))}\right)+\frac{\pi}{2} .
\end{aligned}
$$

We did not find an explicit expression for the dispersion law of hole excitations. Nevertheless, numerical calculation suggests, that the curve shows the expected behaviour (cf. figure 2).

Now we want to derive equations for hole positions and complex roots excluding the vacuum parameters by using the method of Babelon et al. [13]. For this purpose we write down the BAE in integral approximation for a complex root $z$ :

$$
\exp (2 \pi i[I(z)-F(z)])=-1
$$

with

$$
\begin{aligned}
& I^{\prime}(z)=N \int_{-\infty}^{\infty} d \lambda^{\prime} \sigma\left(\lambda^{\prime}\right) \bar{\phi}^{\prime}\left(z-\lambda^{\prime}, \gamma\right) \\
& F^{\prime}(z)=N \phi^{\prime}\left(z, \frac{1}{2} \gamma\right)+N \phi^{\prime}(z, \gamma)+\sum_{h=1}^{N_{h}} \bar{\phi}^{\prime}\left(z-\lambda_{h}, \gamma\right)+\sum_{l=1}^{N_{c}} \phi^{\prime}\left(z-z_{l}, \gamma\right),
\end{aligned}
$$

and $\sigma(\lambda)$ is the regular density

$$
\sigma(\lambda)=\rho(\lambda)+\frac{1}{N} \sum_{h=1}^{N_{h}} \delta\left(\lambda-\lambda_{h}\right) .
$$

$I^{\prime}(z)$ has discontinuities on the lines $\operatorname{Im}(z)= \pm(\pi / 2-\gamma)$. So analysing (4.7) we have to consider three cases. 
a) $\operatorname{Im}\left(z_{l}\right)>\pi / 2-\gamma$. We can evaluate the function $I(z)-F(z)$ directly by integrating

$$
I^{\prime}(z)-F^{\prime}(z)=-\sigma\left(z-\frac{i \pi}{2}\right) N
$$

Noticing

$$
I(\infty)-F(\infty)=0
$$

one gets

$$
I(z)-F(z)=-N \int_{\infty}^{z} \sigma\left(u-\frac{i \pi}{2}\right) d u
$$

and

$$
\exp \left(-2 \pi i N \int_{\infty}^{z} \sigma\left(u-\frac{i \pi}{2}\right) d u\right)=-1
$$

Splitting $\sigma(u)$ into vacuum and excitation contributions

$$
\sigma\left(u-\frac{i \pi}{2}\right)=\sigma_{0}\left(u-\frac{i \pi}{2}\right)+\frac{1}{N} \bar{\sigma}\left(u-\frac{i \pi}{2}\right)
$$

we get for (4.14)

$$
\exp \left(2 \pi i N \int_{\infty}^{z} \sigma_{0}\left(u-\frac{i \pi}{2}\right) d u+2 \pi i \int_{\infty}^{z} \bar{\sigma}\left(u-\frac{i \pi}{2}\right) d u\right)=-1
$$

The first exponent term contains an explicit $N$-dependence, while the second does not. So the validity of the above equation in the limit $N \rightarrow \infty$ requires, that the second term cancels the $N$-dependence in the real part of the first one by approaching $z$ in exponential order towards a pole of the integrand. These poles of $I^{\prime}(u)-F^{\prime}(u)$ are

$$
z_{l} \pm i \gamma, \quad \lambda_{h} \pm\left(\frac{i \pi}{2}-i \gamma\right), \quad \pm \frac{1}{2} i \gamma, \quad \pm i \gamma
$$

with the relevant ones

$$
z_{l} \pm i \gamma \quad \text { with residue } \mp i
$$

Now it can be easily established that

$$
\operatorname{Im}\left(\int_{\infty}^{z} \sigma_{0}\left(u-\frac{i \pi}{2}\right) d u\right)>0
$$

Therefore it must exist $z_{l}$ with $z=z_{l}+i \gamma$.

b) $-\pi / 2+\gamma<\operatorname{Im}\left(z_{l}\right)<\pi / 2-\gamma$. Here evaluating (4.8) by deforming the integration contour provides an additional term due to residue theorem. One has to replace

$$
I(z) \rightarrow I(z)-N \sigma\left(z-\frac{i \pi}{2}+i \gamma\right)
$$


Then (4.7) reads

$$
\exp \left(2 \pi i N \int_{\infty}^{z}\left[\sigma\left(u-\frac{i \pi}{2}\right)-\sigma\left(z-\frac{i \pi}{2}+i \gamma\right)\right] d u\right)=-1
$$

Using the Fourier transforms (4.4) one can establish the relation

$$
\sigma_{0}(u)+\sigma_{0}(u-i \pi+i \gamma)=0 .
$$

Then determining the sign of the real part of the exponent leads to

$$
\operatorname{Im}\left(\int_{\infty}^{z}\left[\sigma_{0}\left(u+\frac{i \pi}{2}\right)+\sigma_{0}\left(u-\frac{i \pi}{2}\right)\right] d u\right)\left\{\begin{array}{lll}
>0 & : & \operatorname{Im}(z)>0 \\
<0 & : & \operatorname{Im}(z)<0
\end{array}\right.
$$

and we conclude that there exists $z_{l}$ with $z=z_{l}+i \gamma$ for $\operatorname{Im}(z)>0$ and $z_{l}$ with $z=z_{l}-i \gamma$ for $\operatorname{Im}(z)<0$ respectively.

c) $\operatorname{Im}\left(z_{l}\right)<-\pi / 2+\gamma$. Evaluating (4.8) again by contour deformation and substituting into (4.7) leads to

$$
\exp \left(2 \pi i N \int_{\infty}^{z}\left[\sigma\left(u-\frac{i \pi}{2}\right)-\sigma\left(z-\frac{i \pi}{2}+i \gamma\right)+\sigma\left(z+\frac{i \pi}{2}-i \gamma\right)\right] d u\right)=-1
$$

Using (4.22) the $N$-dependent exponent term reduces to

$$
2 \pi i N \int_{\infty}^{z} \sigma_{0}\left(u+\frac{i \pi}{2}\right) d u
$$

With

$$
\operatorname{Im}\left(\int_{\infty}^{z} \sigma_{0}\left(u+\frac{i \pi}{2}\right) d u\right)<0
$$

follows $z=z_{l}-i \gamma$, where $z_{l}$ is a BAE root.

Now we are able to determine the configurations in which complex roots can appear. We distinguish three cases for the anisotropy:

a) $0<\gamma<\pi / 4$. Because of symmetry with respect to the real axis we only consider $\operatorname{Im}(z)>0$. This leads to the existence of $z_{l}$ with $z=z_{l}+i \gamma$. Repeating this argument for $z_{l}\left(\operatorname{Im}\left(z_{l}\right)>0\right.$ because of $\left.\gamma<\pi / 4\right)$ generates another root $z_{l}-i \gamma$. This process generating roots breaks down if the last generated root has negative imaginary part. Therefore the 'minimal' configuration induced by $z$ is a chain with spacing $i \gamma$ situated completely in the upper half plane except the last generated root.

For further discussion one has to distinguish wide and close roots.

(i) $\operatorname{Im}(z)>\pi / 2-\gamma$. Taking the product over equations (4.14) and (4.21) respectively for all chain members shows that there remains a non-compensated $N$-dependent term until the chain is continued into the lower region of wide roots. Then the configuration gets 'stable' in the limit $N \rightarrow \infty$. Notice that also the configuration with all roots complex conjugated exists, so that the configuration is actually a double chain. 
(ii) $0<\operatorname{Im}(z)<\pi / 2-\gamma$. Here the above procedure does not lead to a 'stable' configuration. The only way for this configuration to exist in the limit $N \rightarrow \infty$ is arranging the roots symmetrically with respect to the real axis. Therefore this configuration is of the string type. It coincides with its complex conjugated one (see figure 1).

b) $\pi / 4<\gamma<\pi / 3$. and c) $\pi / 3<\gamma<\pi / 2$. Analogous argumentations lead again to short double chains and strings (see figure 1 ).
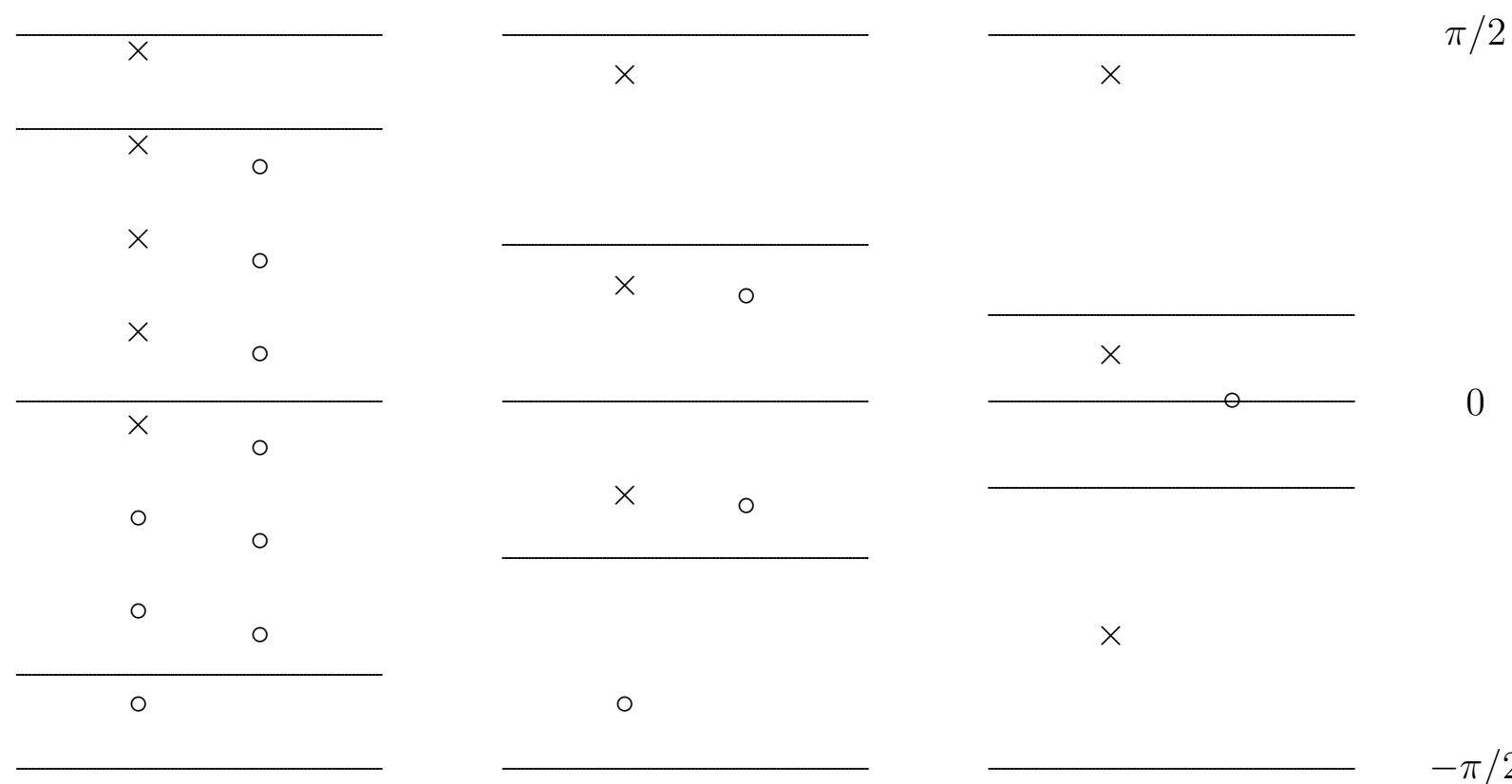

Figure 1. Some special configurations of complex roots for the three cases of $\gamma$. With ' $x$ ' a member of the 'minimal' configuration is symbolized and ' $\circ$ ' denotes additional roots to make the configuration 'stable'. String configuration members are denoted by 'o' too due to the fact that stability is realized by symmetrical arrangement. The lines varying in the three cases denotes $\pm(\pi / 2-\gamma)$. Notice that for non-symmetric configurations the complex conjugated one in this picture is omitted.

We can write down the possible configurations for the three cases in a more compact way:

(i) $z_{t}=z-i t \gamma, t=0 \ldots n, n=\left[\frac{\pi}{\gamma}\right],\left[\frac{\pi}{\gamma}\right]-1, z$ is a wide root

(ii) $z_{t}=z-i t \gamma, t=0 \ldots n, n \leq n_{\max }, n_{\max }=\left[\frac{\pi}{\gamma}\right]-2, \operatorname{Im}(z)=\frac{n \gamma}{2}, z$ is a close root.

The higher level BAE for a state with complex root configurations of the first type can be obtained by taking the product over equations (4.7) for all members and calculating $I(z)-F(z)$ directly via Fourier transforms. With the new parameters

$$
\chi_{l}=z_{l}-\frac{i n_{l} \gamma}{2}, \quad \text { for } n \text { even, }
$$




$$
\begin{aligned}
& \tilde{\chi}_{l}=z_{l}-\frac{i n_{l} \gamma}{2}, \quad \text { for } n \text { odd, } \\
& P \ldots \text { number of } \chi \text {-configurations, } \\
& \tilde{P} \ldots \text { number of } \tilde{\chi} \text {-configurations, } \\
& \alpha=\frac{\pi}{\pi-\gamma}
\end{aligned}
$$

it follows

$$
\begin{aligned}
& \prod_{h=1}^{N_{h}} \frac{\sinh \left(\alpha\left(\chi_{l}-\lambda_{h}\right)+i \alpha \pi / 2\right)}{\sinh \left(\alpha\left(\chi_{l}-\lambda_{h}\right)-i \alpha \pi / 2\right)}=-\prod_{j=1}^{P} \frac{\sinh \left(\alpha\left(\chi_{l}-\chi_{j}\right)+i \alpha \pi\right)}{\sinh \left(\alpha\left(\chi_{l}-\chi_{j}\right)-i \alpha \pi\right)} \prod_{j=1}^{\tilde{P}} \frac{\cosh \left(\alpha\left(\chi_{l}-\tilde{\chi}_{j}\right)+i \alpha \pi\right)}{\cosh \left(\alpha\left(\chi_{l}-\tilde{\chi}_{j}\right)-i \alpha \pi\right)} \\
& \prod_{h=1}^{N_{h}} \frac{\cosh \left(\alpha\left(\tilde{\chi}_{l}-\lambda_{h}\right)+i \alpha \pi / 2\right)}{\cosh \left(\alpha\left(\tilde{\chi}_{l}-\lambda_{h}\right)-i \alpha \pi / 2\right)}=-\prod_{j=1}^{P} \frac{\cosh \left(\alpha\left(\tilde{\chi}_{l}-\chi_{j}\right)+i \alpha \pi\right)}{\cosh \left(\alpha\left(\tilde{\chi}_{l}-\chi_{j}\right)-i \alpha \pi\right)} \prod_{j=1}^{\tilde{P}} \frac{\sinh \left(\alpha\left(\tilde{\chi}_{l}-\tilde{\chi}_{j}\right)+i \alpha \pi\right)}{\sinh \left(\alpha\left(\tilde{\chi}_{l}-\tilde{\chi}_{j}\right)-i \alpha \pi\right)}
\end{aligned}
$$

Calculating energy and momentum for the configurations shows, that for the first type the direct contribution to these magnitudes vanishes. On the other hand there are two hole parameters associated to such a configuration for symmetrical arrangement ('string type') and four for a non-symmetric configuration ('double chain'). So these configurations do carry energy, but only via their associated holes. This is the standard picture, which holds also in our model.

For the second type we get

$$
\begin{array}{r}
\Delta E=-\bar{c} \frac{4 \pi}{\pi-\gamma} \frac{\sin [(n+1) \gamma \pi / 2(\pi-\gamma)] \cosh [\sigma \pi /(\pi-\gamma)]}{\cosh [2 \sigma \pi /(\pi-\gamma)]-\cos [(n+1) \gamma \pi /(\pi-\gamma)]} \\
-\tilde{c} \frac{4 \pi}{\pi-\gamma}\left\{\frac{\sin [(n+2) \gamma \pi / 2(\pi-\gamma)] \cosh [\sigma \pi /(\pi-\gamma)]}{\cosh [2 \sigma \pi /(\pi-\gamma)]-\cos [(n+2) \gamma \pi /(\pi-\gamma)]}\right. \\
\left.+\frac{\sin [n \gamma \pi / 2(\pi-\gamma)] \cosh [\sigma \pi /(\pi-\gamma)]}{\cosh [2 \sigma \pi /(\pi-\gamma)]-\cos [n \gamma \pi /(\pi-\gamma)]}\right\} \\
\Delta P=\sum_{i=0}^{2} \arctan \left(\frac{\sinh [\sigma \pi /(\pi-\gamma)]}{\sin [(n+i) \gamma \pi / 2(\pi-\gamma)]}\right)+\frac{3 \pi}{2}
\end{array}
$$

for $n \geq 1$. The dispersion law of these excitations has been calculated numerically for different $\gamma<\pi / 3$ and string length $n$ and $\bar{c} / \tilde{c}=1$. A comparison with the dispersion law of a free two magnon state, where the momenta of the magnons are equal as given in figure 2 reveals, that these excitations can be identified with bound magnon states in analogy to [13]. One can see from the picture, that the maximum of the curve decreases monotonically with increasing $n$ up to $n_{\max }$ for $\gamma$ fix, approaching the dispersion curve for the free state. On the other hand for $\gamma \rightarrow \pi / 3$, where only the $n=1$ state exists, again the bound state curve approaches the one for the free state monotonically.

Moreover there is a state with $n=0$ allowed through the whole $\gamma$-range. Its energy and momentum are given via (4.29) and (4.30), where in the last formula the term for 
$i=0$ is dropped. This leads to a range for the momentum between those for a single hole excitation and a bound state. Therefore the bound state interpretation fails.
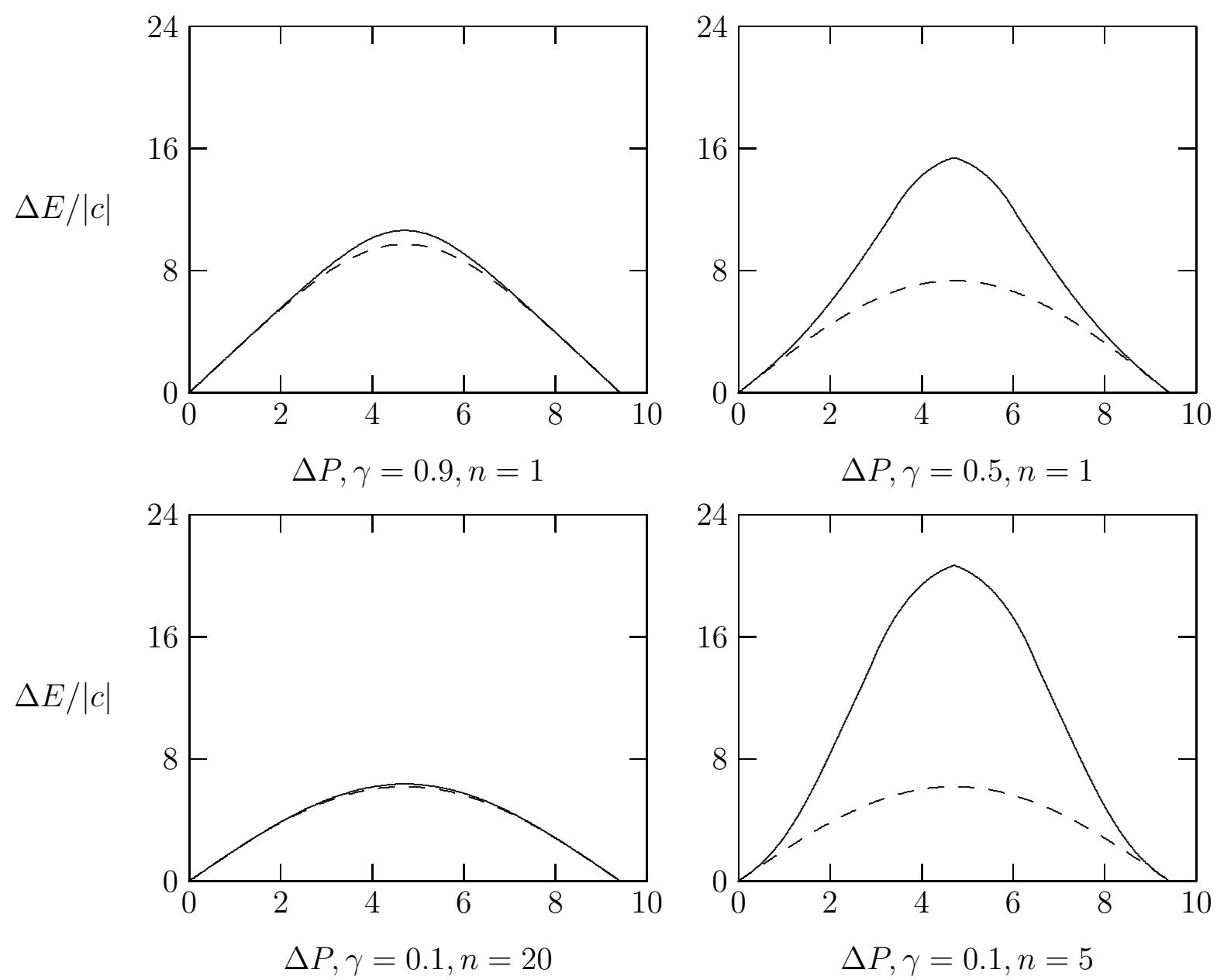

Figure 2. Dispersion relation for Type II excitations (solid line) compared with the dispersion law for a free two magnon state (dotted line) for different $\gamma$.

\section{Conclusions}

We have investigated a generalized Heisenberg spin chain with alternating spins $\mathrm{XXZ}\left(\frac{1}{2}, 1\right)$ in the gapless region. By means of thermodynamic Bethe ansatz (TBA) integral equations determining the ground state have been derived. In the case of negative coupling of spin interactions these equations were solved. It turns out that the ground state is formed by a sea of $(1,-)$ - strings and is therefore of antiferromagnetic nature.

Weakly excited states above this antiferromagnetic vacuum have been analysed following the method introduced in [13]. In analogy to the XXZ $\left(\frac{1}{2}\right)$ model for 
$-1<\Delta<0$ two types of excitations appear. The first one are scattering states of magnons. Higher level BAE are derived which determine the parameters of these excitations. The second type can be identified with bound magnon states in analogy to [13].

\section{Acknowledgment}

We would like to thank H. M. Babujian for helpful discussions.

\section{References}

[1] de Vega H J and Woynarovich F 1992 J. Phys. A: Math. Gen. 254499

[2] Faddeev L D and Takhtajan L A 1979 Usp. Math. Nauk 345 [Russ. Math. Surveys 34 (1979) 11]

[3] Kulish P P and Sklyanin E K 1981 Lecture Notes in Physics vol 151 (Berlin: Springer) 61

[4] Takhtajan L A 1982 Phys. Lett. 87A 479

[5] Babujian H M 1983 Nucl. Phys. B215 [FS7] 317

[6] Babujian H M and Tsvelick A M 1986 Nucl. Phys. B265 [FS15] 24

[7] Takahashi M and Suzuki M 1972 Progr. Theor. Phys. 482187

[8] Korepin V E 1979 Theor. Math. Phys. 41953

[9] Tsvelick A M and Wiegmann P B 1983 Adv. in Phys. 32453

[10] Kirillov A N and Reshetikhin N Yu 1985 Zap. Nauch. Semin. LOMI 145109 [J. Sov. Math. 35 (1985) 109];

Kirillov A N and Reshetikhin N Yu 1985 Zap. Nauch. Semin. LOMI 14631 [J. Sov. Math. 40 (1988) 22];

Kirillov A N and Reshetikhin N Yu 1985 Zap. Nauch. Semin. LOMI 14647 [J. Sov. Math. 40 (1988) 35]

[11] Kirillov A N and Reshetikhin N Yu 1987 J. Phys. A: Math. Gen. 20 1565;

Kirillov A N and Reshetikhin N Yu 1987 J. Phys. A: Math. Gen. 201587

[12] Frahm H, Yu N C and Fowler M 1990 Nucl. Phys. B336 (1990) 396

[13] Babelon O, de Vega H J and Viallet C M 1983 Nucl. Phys. B220 [FS8] 13

[14] de Vega H J, Mezincescu L and Nepomechie R I 1994 Phys. Rev B49 13223

[15] de Vega H J, Mezincescu L and Nepomechie R I 1994 Int. J. Mod. Phys. B8 3473

[16] Aladim S R and Martins M J 1993 J. Phys. A: Math. Gen. 26 L529

[17] Aladim S R and Martins M J 1993 J. Phys. A: Math. Gen. 267301

[18] Martins M J 1993 J. Phys. A: Math. Gen. 267287 


\section{Figure captions}

Figure 1. Some special configurations of complex roots for the three cases of $\gamma$. With ' $x$ ' a member of the 'minimal' configuration is symbolized and 'o' denotes additional roots to make the configuration 'stable'. String configuration members are denoted by ' $\circ$ ' too due to the fact that stability is realized by symmetrical arrangement. The lines varying in the three cases denotes $\pm(\pi / 2-\gamma)$. Notice that for non-symmetric configurations the complex conjugated one in this picture is omitted.

Figure 2. Dispersion relation for Type II excitations (solid line) compared with the dispersion law for a free two magnon state (dotted line) for different $\gamma$. 